\title{
CONSULTING CHESS WITH A COMPUTER - A SEQUEL
}

\author{
M. Valvo \\ Edison, New Jersey \\ USA
}

[In a previous issue of the Journal (Vol. 13, No. 2, pp. 88-98) a report was published on the first game between the Valvo Team (Mike Valvo and Belle, assisted by Ken Thompson) and the Bono Team (a team of 2400USCF-rated players from Boston), played in correspondence style over the UNIX net. The game was finally lost by the Valvo Team after a refused draw. - Eds.]

\section{Game 2}

\section{Bono Team - Valvo Team}

\section{1. e4 e5 2. Bc4 Nf6}

This 2. Bc4 line is becoming popular again (Nunn, Kudrin). It is trickier than it seems on the surface. Most who play this move order will play 3. d3 now, but 3. Nc3, aiming for a Vienna, is also possible. 3 . d3 d5?! 4. ed Nd5 5. Nf3 Nc6 6. 0-0 is much in White's favour because of Black's weak e-Pawn. This stuff requires much patience on Black's part and the best he can hope for is a nagging slight disadvantage.

\section{3. d3 Nc6}

This opening has been relatively popular over the past ten years for second-level world-class players (Nunn, Kudrin, Dolmatov, Larsen, Torre) when playing weaker players or when content with a draw. The two famous Nunn-Korchnoi games in this line are an exception because Nunn didn't trust the white side of the Ruy Lopez at that time. In all the lines White can choose, the resulting pawn structure and ensueing strategies are very similar to closed Ruy Lopezes. Black can choose to put his King's Bishop at e7 and c5. Some black players when facing this opening for the first few times play an early $c 6$ and transpose into a Philidor Defense. Black has to be careful about playing d5. If it is played too early, e5 can be permanently weakened. On the other hand, it is often the equalizer. With the move order I have chosen, I have pretty much committed my team to moving my f8-Bishop or 4. ... h6 if White now plays 4. Nf3 (threat $\mathrm{Ng} 5$ ). I intend to try to save moves on a Bg7 strategy by avoiding Be7-f8-g7 and try to get $\mathrm{Bg} 7$ in at once: 4. Nf3 h6 5. 0-0 g6 6. c3 Bg7 7. d4 0-0. Other black choices were:

a) 3. ... Bc5 4. Nf3 d6 5. 0-0 Nc6 6. c3 a6 7. Bb3 Ba7 8. Nbd2 0-0 9. Re1 Be6=(Nunn-Smejkal 1982). Black actually won.

b) 3. ... c6 4. Nf3 Be7 5. 0-0 d6 as played by Ivanchuk several times (also Torre when he played Black).

c) 3. ... Be7 4. Nf3 d6 5. 0-0 0-0 =/+=.

\section{Nc3 Na5 5. Nf3 d6}

White's 5. Nf3 was a mild surprise as most lovers of this line play 5. Nge2. After White's fifth, Black has to think about the placement of his f8-Bishop. The choices, as I see it, are b4, e7 and g7. Knaak once (1974?) played 5. ... Nc4 6. dc Bb4 7. Qd3 d6 8. Bd2 0-0 9. 0-0-0 unclear. Most blacks intend Be7, but I am wondering about g6 and Bg7. Psakhis played this way against 5. Nge2. In any case, Black's plan is an eventual f5. If Black goes the $\mathrm{Be} 7$ route, he will try to exchange Bishops with $\mathrm{Bg} 5$.

\section{Bg5 $\mathrm{Be} 7$}

This has been played before with the continuation being 7. Bf6 Bf6 8 . Nd5=. White has to be satisfied with an almost non-existent edge. This edge consists mainly of a little initiative and the fact his pieces are poised more aggressively. Black's position is solid and it contains no weaknesses. Black still has hopes of f7-f5 at some point, while White will aim for a kingside attack. White's other sixth move try is $6 . \mathrm{h} 3$ intending $7 . \mathrm{g} 4$.

\section{7. h3 0-0 8. Qd2 c6}

This (8. Qd2) is new. 8. Qe2 was played previously in a game of Nunn's (8. Qe2 c6 9. Bb3 h6 10. Bd2 Nd7 11. Bf7 Rf7 12. b4 Nf8 with an edge to Black). I think White's best is 9. Bb3. After the text c6, Black has ideas of both ... b5 and ... d5. Right now Black threatens 9. ... Nc4 10. dc h6 forcing a bishop-for-knight trade: 11. Bh4? $\mathrm{Ne} 4$ ! or 11. Be3 Ne4! 12. Ne4 f5. A possible sequence is 9. a3 Nc4 10. dc h6 11. Bf6 Bf6 12. 0-0-0 Be6! White 
is unlikely to castle Queen's side because there is too much danger to his King. King's side castling is not attractive either because Black can aim for f5 effectively. Note that 8. Qd2 removes the indirect control of the d1h5 diagonal so Black has the additional possibility of ... Nh5 in the future.

\section{Bb3 h6}

I think Black has already equalized and may be just a hair better. 10. Bf6 Bf6 is clearly fine for Black. 10. Bh4 Ne4! poses no problems either: 11. Be7 Nd2 12. Bd8 Nf3 or 11. Ne4 Nb3 12. ab Bh4 13. Nh4 Qh4 14. Nd6 a6 or 11. de Nb3 12. ab Bh4 13. Nh4 Qh4 14. Qd6 Qg5. They probably have been relying on 10. Be3, but 10.... $\mathrm{d} 5$ ! threatening to fork two pieces is a killer. For example, 11. ed cd 12. $\mathrm{d} 4 \mathrm{Bb} 4$ ! What has happened here? White has seemingly made no mistakes. He has developed all his pieces to effective squares and is ready to castle. I feel White made a strategic error by playing Nc3. Instead he should aim for $\mathrm{c} 3$. Still, he was by no means worse at that point. Perhaps the major problem was Nf3 instead of Nge2. Now let me explain what I am trying to communicate: White's pieces, while well developed, have no real impact on the position. The main problem is Black, by playing a timely ... $\mathrm{c} 6$, has turned the battle for the center into one of Pawns versus pieces. White has placed his pieces in front of his Pawns while Black did the opposite. When the Pawns eventually advance, the pieces must give way. Strategically, White would want to challenge the black pawn structure with $\mathrm{d} 4$, but his hanging Bishop prevented that. In addition, Black can win White's Bishop for a Knight whenever it is appropriate. Finally, Black has been careful not to create weaknesses. White's singular plan has been a kingside storm against Black's King. But it never got going. Meanwhile his pieces look awkwardly placed. With all this, Black has only a very minimal edge. It will, when things get sorted out, consist of a slightly better pawn structure and a whisp of an initiative.

\section{Bxf6 Bxf6 11. 0-0 Nxb3}

Interesting. White concedes the fact that Black has the edge without making Black fight to prove it. It is not clear to me that Black could claim much of an advantage after 10. Bh4 Ne4 11. Ne4 Nb3 12. ab Bh4 13. Nh4 Qh4 14. Nd6. It would be clear that if anyone would be better, Black would be. 10. Bf6 seems to both concede the advantage and allow Black the scope to convert it into something more tangible. Anyway, back to the position at hand. White is now in a position (after 11. 0-0) to play d4. While that is not a danger in itself, it would neutralize any temporary advantage in initiative I have. Black has two possible plans as I see it: (1) play d5, Qd6, Re8 and control the center. White's counter piay would be minimal. Then go for $\mathrm{Kh} 7, \mathrm{~g} 6, \mathrm{Bg} 7$, f5; or (b) go directly for $\mathrm{Kh} 7, \mathrm{~g} 6, \mathrm{Bg} 7$, f5. But the latter plan would allow a white $\mathrm{d} 4$. I decided to stall for one move by capturing the b3-Bishop. Ken has already left for Edmonton and the World Championship for Computer Chess and I have been playing without Belle for the past two moves. I decided I wanted to confer with her before playing my intended $12 . . . \mathrm{d} 5$.

What is the black advantage? (1) The obvious two Bishops (2) A flexible pawn structure (3) The initiative. I wonder if Deep Thought would have played 10. Bf6 or chose to slug it out in one of the other lines.

\section{2. $\operatorname{axb3} \mathrm{d} 5$}

Black has a problem in that he has an inherently defensive posture and the advantage has been thrust upon him. While he is not happy about playing $12 \ldots \mathrm{d} 5$, he doesn't want white counterplay after (say) 12.... Qe7 13. d4. I presume White will play 13. Rfe1 and I must play 13... Re8 (13... Qd6!? 14. d4! ed 15. Nd5!=). White could then try 13. Rfe1 Re8 14. ed cd 15. d4 e4 16. Ne5. Belle thinks that Black's advantage is on the order of $1 / 3$ of a Pawn, but that might be the pawn structure plus the two Bishops.

\section{3. exd5 cxd5 14. Rfe1 Re8}

I suppose White didn't want to give Black the opportunity to play d5-d4 and that is why the early capture 13 . ed. Black lags in development, but Black controls the center at the moment. I have been assuming that White will now play 15 . $\mathrm{d} 4$, but maybe 15 . Re2 is possible. That would pose no problem as 15 . Re2 a6 16 . Rae1 Qd6 is fine for Black.

\section{Re2 Qd6}

The computer expected 15. Re 2 as White's best. On general considerations, however, I am not sure what they are doing because one wouldn't make an exchange like 13. ed if not intending an immediate $\mathrm{d} 4$ to challenge the center. If Black is allowed the two Bishops and a fluid center, the future is bleak for White. Black had three reasonable choices at this juncture: $15 . . . \mathrm{a} 6,15 \ldots$... Bf5 and $15 . .$. Qd6. The first two are aimed at simple development. The third is Belle's judgement which I like very much. It is a good idea to not disturb Black's queenside pawn structure because White might be able to maneuver a Knight to the b6-square. Note that while $15 . .$. Qd6 does nothing to prevent a resource available at White's last turn: 15. d4 e4 16. Ne5 Be5 17. de Re5 18. Re4!, it does maintain a flexible posture. One positive virtue of 15.... Qd6 is a subsequent Bd7 protecting the eRook becomes a harmonious possibility. 


\section{Nb5 Qc5}

Belle now thinks our last move (15. ... Qd6) was a serious mistake that dissipated our entire advantage. Belle 'knew' that 16. ... Qb6? would be met by 17. Qa5!, but she thought that $16 . . . \mathrm{Qb8}$ would be adequate. Poor Belle had her associative memory turned off because Ken was trying to isolate a hardware bug and she didn't see that White could simply draw by playing back to $c 3: 15$. Nb5 Qb8 16. Nc3. Worse, White could play for more with 16. c4!? For better or worse, 16.... Qc5 is forced. Black probably should have played 15 ... Bf5 as we have now lost the initiative and control of the game. White has considerable counterplay chances (attacking chances!?) with ideas like Ra5 or Qa5. White also has possibilities with an eventual d4 because Black's e-Pawn is pinned to the e8-Rook. Black's chances lie in a timely e5-e4. Attacks on the a5-square with White's Queen or Rook can be repulsed with Bd8. Probably White's most dangerous chances lie in 17. c4!? This (a) protects the Knight, (b) attempts to break up Black's center, (c) fights to control the d5-square for a Knight. Black has two plans of reaction: 17... Be6 or an immediate 17. ... e4. Even though Black has development problems, they both give sufficient chances. A fantasy line we looked at was 17. c4 e4 18. de de 19. b4 Qh5!? 20. Nc7 Rd8 21. Qe1 Bh3!! winning. We also looked at very complex lines like 17. c4 Be6 18. Qa5 Bd8 and 18. ... e4!? Black no longer has an advantage. White's Passive-Aggressive style lulled us into thinking they were going to continue to be lambs awaiting the slaughter. There is a silver lining, though, to all this. In order to create confusion and generate chances for himself, White has to completely open the position. In many lines it appears like Black is just holding everything together when suddenly the Bishops assert themselves and come into their own.

17. c4 Be6

Things are now getting wild. White has various moves, but now he must settle on a plan. 18 . b4 seems senseless after 18. ... Qb6. 18. Qa5 is dangerous and Black will have to choose between 18.... Bd8 and 18.... e4 (e.g. 18. Qa5 e4 19. de dc). Strategically, White is trying to break up Black's center. He would also like to be able to use the d5-square as an outpost for his Knight. Black has a problem with his vulnerable e-Pawn. He has a trump in that he can exploit the dynamicism of his center duo by trying ... e4!? at some point.

18. Qe3 d4

This (18. Qe3) was somewhat unexpected and it is very strong. We spent time looking at 18. Qa5 and 18. Rae1! 18. Qe3 has pluses and minuses. It forces Black (otherwise Black loses a Pawn) to block the position and creates a temporary hole on e4, but it denies the c3-square to the b5-Knight. White's best is 19. Qe4 and Black again has problems. 19. ... a6!? is met by 20 . Qb7!, while $19 . .$. Qb6 is met by $20 . \mathrm{Ne} 5 \mathrm{Bh} 321$. f4! winning a Pawn.

\section{Qd2 a6}

White's 19. Qd2 is not best as Black again has a solid advantage. 19. Qe4 was the most challenging and the computer thought that after 19.... a6 20. Qb7! Reb8 21. Qe4 Bd7 22. Na3 Bc6 23. Qf5 Bf3 it could equalize.

Interesting is 19. Qe4 a6 20. Qb7 Bf5!? with tremendous counterplay with the central Pawns. But I had something else in mind: 19. Qe4 a6 20. Qb7 e4!!? 21. Qe4! (21. Re4 or 21. de lose a piece to 21. ... Reb8 22. Qc7 $\mathrm{Rb} 5$ !) Bd7! 23. Qb7 Bc6 24. Re8 Re8 25. Qa6 Bf3 26. gf Qg5+ with a tremendous attack. Black sacrifices several Pawns in return for chances against the white King. But Belle thinks it is all a draw after 27. Kh1! Belle is now happy .3 Pawns worth. The game will continue $20 . \mathrm{Na} 3 \mathrm{Bd} 7$ and Black has excellent prospects.

20. Na3 Bd7

Black's plan is simply to embarrass the f3-Knight with 21 ... Bc6. Subsequently, Black will try for $\mathrm{f} 5$ by considering a plan of Kh7 (or h5), g6, Bg7 and f5. Note that 21. b4 Qc7 (21... Qc6!? threatening 22... Bh3) 22. b5 drops a Pawn to 22.... ab 23. cb Bb5.

21. Qe1 Qc7

White now has several good plans. The obvious one is Nd2, Ne4. A second is b4, c5, Nc4, Nd6. A third less likely possibility is $\mathrm{Nc2}, \mathrm{Nb} 4$ and Nd5. Black's goal is to play single mindedly for f5. Black may take time out to play $\mathrm{b} 5$ at some point to deny White his knight access to $c 4$. The purpose of 21 .... Qc7 is to reposition her before playing Bc6. This flexible defense makes it difficult for White to proceed. In order to play for a win, Black will have to play $\mathrm{f} 5$ and $\mathrm{e} 4$ at some point. White will defend by playing $\mathrm{Nd} 2$ and, if necessary, $\mathrm{f} 3$. It is not yet clear how Black will break through. In the meantime, White has nothing better to do but defend or try the b4-c5 \& N-c4-d6 ideas.

\section{Nd2 Qb6}

White's 22 . Nd2 was a surprise. We expected 22. b4 and we were considering 22. ... Bc6 23. Nd2 Bg5!? with the idea 24. Ne4 Be4! and Black can force e4: 25. Re4 (25. de? d3 wins the exchange) f5 26. Re2 e4! and 27. de? would lose the exchange. The played 22.... Qb6 is a prophylactic in nature. Since White neglected to play b4, Black is going to hold that down and focus on it as a weakness. Although normally one doesn't want to move a piece twice like this, this seems justified. White has relieved the pressure on the e-Pawn and the Queen 
now has the possibility of going to g6 in one move. Without b4 White's position will drift into passivity. Black will work on 55 and 44 while White can only defend.

\section{Ne4 Be7}

White has problems. Right now his b-Pawn is unprotected and Black threarens to push his Pawn to f5. 24. Nd2 runs into 24. ... Bb4. I have no idea how White expects to untie his position.

\section{4. c5 Bxc5}

White's last pretty much forces an endgame (24.... Bc5 25. Nc4 Qc7 26. Nc5 Qc5 27. Re5 Re5 28. Qe5 Qe5 29. $\mathrm{Ne} 5 \mathrm{Bf5}$ ) that is significantly in Black's favor. Other tries are risky and even losing for Black. For example, 24. ... Qb3!? 25. Nc4 f6 26. Ra3 and White will win the exchange: 26. ... Qb5 27. Ncd6 Bd6 28. Nd6 Qc5 29. Ne8 $\mathrm{Re} 8$ 30. f4 Bc6 with rough equality. Dangerous is 24 . ... Qg6 25. Nc4 Bh3 26. Ng3. 24. ... Qe6 seems to give Black the best chances of maintaining an edge, but it is an illusion. The idea is to play for activity along the c6g2 diagonal and protect the e5-Pawn in anticipation of Nc4: 24. ... Qe6!? 25. Nc4 Bc6 26. Ncd6 Bd6 27. Nd6 Qd5! 28. f3 Re6 29. b4 Rf8 30. Nc4 Rfe8. However, White can obtain a winning game with 24. ....Qe6 25. Nc4 Bc6 26. $\mathrm{f} 4$ !

Belle suggested the idea of 22. ... Qb6, and I liked it. I must admit I didn't do more than look at the current position at the time, and if I had considered the text line in more detail, I would not have been happy after 24. c5!? However, $95 \%$ of our time was spent on the other game, and I was happy with Belle's proposals. When you play on a team with a computer, you know they look at everything (at least superficially) even though the line does not come up in discussion. Believe me, this factor does not promote good habits in the human side of the team.

The endgame they are forcing is not good for them, but it is much better than the current middlegame position they have. I feel confident we can win it due to the weak white Pawns and the fact we have a bishop-versusknight endgame with Pawns on both sides of the board. But there is no longer the possibility of a crush.

\section{Nc4 Qb4 26. Nxc5 Qxc5 27. Nxe5 Bf5 28. Rc1 Qb5}

This was expected, although White could have also tried the weakening 28. g4!? The computer now thinks 29 . b4!? Rec8! 30. Rec2 Rc2 31. Rc2 Bd3 nets a Pawn for Black.

\section{9. b4 Re6}

This game has become interesting (especially since we no longer feel we have winning chances in game one!). White has several trappy ideas revolving around the fact that e8 is weak and a timely Nc6! can cut communication between the Queen and e8-Rook. Our main two candidate moves are 29. ... Rec8 and 29. ... Re6! 29. ... Rac8 looks attractive, but 30. Rc4! and Black has nothing: 30. Rc4 Bd3?? 31. Nd3! Re2 32. Rc8+ wins or 30. Rc4 Rc4 31. dc Qa4 32. c5 unclear (32.... d3? 33. Nd3!).

We like both candidate tries, but 29. ... Re6 seems to net a Pawn: 29. ... Re6 30. Nf3 Qd3 31. Re6 Be6 32. Rd1 Qg6! 33. Ne5 Qc2 34. Rd2 Qb3 -=. Or 29. ... Re6 30. g4!? Bh7! 31. f4!? Qe8 with f6 to follow.

\section{44 Bh7}

White finally weakens his king position. I am trying to convince Ken that if $31 . \mathrm{f} 4$, we should continue 31 .... Bd3!? 32. Nd3 Qd3 33. Re6 fe 34. Qe6 Kh7 and the extreme weakness around White's King will tell. If 31 .... $\mathrm{Bd} 3$ 32. Rc5 Be2! 33. Rb5 Bb5 looks good. If instead 31. Rc5 Qe8 with f6 to follow.

\section{Rc5 Qe8}

In view of f6, White has no choice but to play 32. Rcc2. The idea is to play 32... Qe7 33. Nf3 Bd3 34. Re6 Qe6 35. Qe6 (35. Rd2!?) fe 36. Rd2 Be4 37. Nd4 Rd8 and Black can force a favorable B vs. N endgame because of the pending e5. Also possible is 32. Rcc2 Qa4!? 33. Nf3 and now the simple Re2 looks strong (not 33.... Qc2 34. Rc2 Re1 35. Ne1) as 34. Re2 Bd3 35. Rd2 Re8 36. Qc1 (36. Qd1? Qd1 37. Rd1 Be2) Be4 37. Nd4 Qb4 drops a Pawn.

\section{Rec2 Qa4}

We sent a long 'if-move' at this point: if 33. Nf3 Bd3 34. Re6 Qc2 35. Re8 Re8 36. Qe8 Kh7. 33. Nf3 looks forced due to the threat of Rae8. White can also vary with 35. Nd4, but 35. ... Qb2 36. Rd6 Rc8 looks very strong. We feel we will come out of all this with a minimum of one Pawn advantage.

\section{3. f4 f6}

White's 33. 44 was unexpected, but it turns out to be very good. We don't appear to be winning a Pawn as we originally thought. 33. ... f6 seems best, although other ideas came into consideration. White threatens 34 . f5 when $34 . . . \operatorname{Re} 5$ is not possible due to a mate, but 34 ... Bf5 first comes into consideration (to create a hole on $\mathrm{h} 7$ for the King). The problem then is the fact the black Queen is not protected: 33. ... Qb3?! 34. f5! Bf5 35. Qd1! (it is true that if Black passed on his $33^{\text {rd }}$ turn, 36... Qe8 would be fine). The threat is not so much $\mathrm{f5}$ as 34. Rc7 and f5 next. After 33... f6 34. Nf3, Black has three methods of continuation: (a) 34 ... Re2 (b) $34 . \ldots$ $\mathrm{Bd} 3$ and (c) $34 . .$. Re3!? I favor (a) while the computer prefers (b). After 34... Re2 (1) 35. Qe2!? Qb4 [35.... 
Re8!?] 36. Rc4 Qb6 gives Black some initiative and (2) 35. Re2 Bd3 36. Re7 Bg6 37. Nd4 Re8 38. Nf5 Bf7 [38. ... Kf8!?] permits Black to retain his advantage of the doubled Pawn in return for some temporary initiative. If instead 34. ... Bd3 35. Re6 Qc2 36. Nd4 Qb2 with severe complications. Finally, 34. ... Re3!? 35. Nd4! Rd3 36. Ne6 is unclear.

\section{Nf3 Bxd3}

This was accompanied with an if-move: if 35 . Re6 Qc2. After that White can try 36 . Re7 or 36. Nd4. They seem to mostly transpose into each other with one major exception. First, the main line: 34. ... Bd3 35. Re6 Qc2 36. Re7. At his point there are a number of possibilities: (a) $36 . .$. Qb2? 37. Rg7+ with a perpetual (b) 36. ... Bg6? 37. Qe6+ Kh8 38. f5 with a big edge (c) $36 . . . \mathrm{Kf8}$ (d) $36 . .$. Kh8. Alternatively, on $36 . \mathrm{Nd} 4 \mathrm{Qb2} 237$. Rd6, Black can try 37... Bg6 intending Bf7 at some point. There are lines where White gets his Rook on the Seventh and Knight on e6 and Belle thinks its overextended due to his weak kingside Pawns.

\section{Rxe6 Qxc2 36. Re7 Kh8}

This incredible move wins a Pawn. White has no constructive reply as Black merely threatens $37 . . . \mathrm{Qb2}$. If Black had played 36. ... Qb2, then 37 . Rg7! draws. 37 . $\operatorname{Rg} 7$ now is not possible because of 37 ... Be4! winning. Belle (selective-search version) had this in mind all the way back to that long if-move we gave. The 'if' line was actually best for White as he could get out without losing any material. Now White's entire king side remains weak and will eventually fall bit by bit. In retrospect we wondered about the psychological aspects of offering an 'if' move with the best variation for your opponent. They are unlikely to believe you! We simply (and naively) send the best line according to the computer. Why didn't we see the Pawn win all along? It was detected by the long-range selective searches. We make short-range moves by brute force (at most 12 ply; usually less). It wasn't until we got to this position that the pawn win was within range of brute-force searches.

\section{7. f5 Rg8}

I had to overrule the computer on this one. Belle wanted to play $37 . .$. Bc4?, but White draws easily with 38 . Nd4 Qb2 39. Re8+ Re8 40. Qe8 Bg8 41. Ne6 Qb4 42. Qd7=. The text is the only move to maintain Black's chances. If now 38. Re8 Bc4 39. Nd4 Qd3!

\section{Qf2 Rd8}

Now if White tries 39. Qe1 Qb1 offers real winning chances. Black will now fight to keep the d-Pawn even if it means giving up the b7-Pawn. This will force White to block on $\mathrm{d} 2$ at some point. Black will have options of play on the king side (h5, Kh7-h6), repositioning his Bishop (b5 or maybe c6) and finally playing Rc8 or e8 to penetrate the white position.

\section{Qe1 Qb1}

It seems to us that White will now agree to exchange Queens. If 40. Rb7?? Qe1+41. Ne1 Be4! 42. Re7 d3 wins a piece. A possible continuation from this position is $40 . \mathrm{Qb1} \mathrm{Bb} 141$. Nd2 $\mathrm{Bd} 342 . \mathrm{Rb} 7 \mathrm{Bb} 543$. $\operatorname{Re} 7 \mathrm{Rc} 8$.

\section{Qxb1 Bxb1 41. Kf2 Ba2 42. Ke1 Kh7}

White's 42 . Kel was a surprise. Belle thought 42 . Re4 was only a slight negative. Now she is happy by about 1.15 Pawns worth. Belle thought there were three tries in the position: (a) $42 . . . \mathrm{Kg} 8$ (b) $42 \ldots \mathrm{Kh} 7$ and (c) 42 . ... h5. Belle liked the text the best. If now 43. Kd2?! Bc4! (threat Bf1) or 43. Re4 d3 44. Kd2 h5!

\section{3. h4 h5}

Belle immediately wanted to play $\mathrm{Kg} 8$ and I thought that was reasonable, but an h5 by them could effectively seal off the king side and their $\mathrm{g} 4$ weakness looked hard to get at. Belle's value jumped from 1.15 Pawns to 1.5 Pawns after 43. h4. The problem with it begins to take shape after 43.... h5! (1) The f5-Pawn becomes undermined and vulnerable to an eventual Bbl (2) The h4-square is no longer available to the Knight. 44 . g5 looks forced, whereupon we will play 44. ... Kg8! Then 45. g6 Kf8 46. Re4 Bb1 47. Rd4 Re8+ 48. Kf2 Bf5. Or 45. gf gf 46. Re4 Bb1 47. Rd4 Re8+48. Kf2 Bf5 with a solid instead of a vulnerable pawn edge.

\section{4. $95 \mathrm{Kg} 8$}

The selective search version of Belle thinks we will win another Pawn soon. Almost certainly, 45. gf gf will be played. If $45 . \mathrm{g} 6 \mathrm{Kf} 8$ ! is too strong as a subsequent $\mathrm{Bb} 1$ will win the vulnerable White $\mathrm{f} 5$ and g6 Pawns.

45. g6 Kf8 46. Re4 Bd5

While two moves have gone by here ..., the situation is much more complex. White chose to play 45 . g6 as opposed to 45 . gf. This could be much more serious for them because if the f5-Pawn goes, the g6-Pawn will surely soon follow. We, however, have to deal with the fact two winning lines stare us in the face: (1) 46. .. Bd5 (Belle's preference) 47. Rd4 Re8+ 48. Kf2 Be4 49. Rd7 Bf5 50. Rb7 Bg6 51. b5 ab 52. Rb5 and Black has excellent winning chances (2) 46. ... Bbl (my preference) 47. Rf4 Re8+ 48. Kf2 Re4 49. Re4 Be4 50. Nd4 Ke7 and Black gives up his extra Pawn in return for tieing White up. What doesn't work is 48. ... Rd5!? 49. Nd4 Ke7 thinking White is completely tied up. Black hopes to get his King to d5 and that the White position will quickly 
collapse from the strain. Belle obviously couldn't envision such a plan. However, the plan doesn't seem to work! White plays 50. Kf2 Kd6 51. Kg3 threatening 52. Ne6! Bf5 53. Ng7=.

\section{Rxd4 Re8+ 48. Kf2 Be4}

We expect play will continue $50 . \operatorname{Rd} 7$ Bf5 51. Rb7 Bg6 52. b5 and now either 52... ab 53. Rb5 or 52... Be4 53. Rb6 ab (53.... a6?!) 54. Rb5 Bf3 (54 .... g6!?) 55. Kf3 g6. Black looks to be winning now.

\section{Rd7 Bxf5 50. Rxb7 Bxg6}

White will likely play 51 . b5 to try to create Q-side counterplay. Black will look to exchange off the minor pieces to prevent any attempt from White to sacrifice the Knight for the remaining black Pawns.

\section{1. b5 Be4}

Black, after the forced 52. Rb6, has to decide between the rational 52. ... ab 53. Rb5 g6 which must be a technical win and the speculative 52 .... a5?! to try to take advantage of the the tactical potential in the position.

\section{Rb6 axb}

We have decided on 53. Rb5 g6 (54. Nd2? Bd3). So we sent the if-move 53. Rb5 g6. Black will work to play g5 (i.e. $\mathrm{Kf} 7, \mathrm{Re} 7, \mathrm{Kg} 7, \mathrm{Re} 6, \mathrm{Kh} 6, \mathrm{~g} 5$ among other similar ideas) and win on the king side. 53 . Rb5 Bf3 54. Kf3 g6 is also possible, but the possibility of a Rook and BP\&RP versus Rook endgame seemed too probable. There's no rush as White will never queen his b-Pawn without help from his King or Knight.

\section{Rxb5 g6 54. Nd4 Bh1}

Probably most plans for Black will eventually win. The goal is not to win the fastest, but the surest or safest. However, they are forcing the game to a crisis by arranging pieces to escort their b-Pawn forward. Hence, we have decided to play aggressively. Black has many plans in the position (Re7, Kf7). The point of 54 ... Bh1 is to vacate the e4-square so the Rook can occupy it. Black wants to threaten to get at the weak white h4-Pawn partly to try to win it and partly to distract White from his queenside counterplay. If White tries to ignore the threat by playing 55 . Rb6 expecting $55 . . . \mathrm{Kf} 7$, Black will cross him up with $55 . . . \mathrm{Re} 4$ ! anyway. White then comes close to a draw by giving up his Knight, but doesn't quite make it: 55. Rb6 Re4! 56. Rf6?! Ke7 57. Rg6 Rd4 58. Rg5 Rd5!! winning (not 58. ... Rh4 59. Kg3!=). If, instead, 55. Nf3, Black can play 55. ... Re4! anyway and White must lose some material. Probably best is 55. Rb4!? planning to meet 55 ... Re4 with $56 . \mathrm{Kg} 1$ ! and the h4-Pawn is immune due to the threat of 57. Ne4+ discovering on the Rook along the fourth rank. But Black then can simply play $57 . . . \mathrm{Kf} 7$ intending $58 . . . \mathrm{Bf} 3$ and $59 \ldots \mathrm{g} 5$.

\section{Kg1 Re1}

An interesting defense! If $55 . . . \mathrm{Re} 156 . \mathrm{Kh} 2 \mathrm{Re} 4$ or $55 . . . \mathrm{Re} 4$, they intend Nf5! (Rb4!?) to hold things tactically. The computer thinks both of White's legal replies are reasonable. I personally expect $55 . . . \operatorname{Re} 156 . \mathrm{Kh} 2$ when we will probably just consolidate with 56 ... Kf7 (or e7 or g7). 56... Be4!? is also interesting with further ideas of $\mathrm{Rh} 1+, \mathrm{Rg} 1+$ and $\mathrm{Rg} 4$. The computer doesn't understand that RP+BP endings are often drawn with only Rooks on the board, so that complicates using it to analyze such positions.

\section{Kh2 Be4}

The computer has finally come around to my way of thinking on this move and nearly sees a win of another Pawn. Black threatens 57. ... Rh1 $+58 . \mathrm{Kg} 3 \mathrm{Rg} 1$ followed by Rg4 winning the white h-Pawn. The computer isn't totally clear about 57 . Ne6+ (so I could say the h4-Pawn is definitely lost), but I think it is only a horizon problem.

\section{Ne6+ Ke7 58. Nc5 Bc6}

White clearly has to keep his Rook on the b-line to protect b2, but where? Belle likes 59. Rb6 Kc6!? (I prefer the natural 59... Bd5). White seems to only be able to keep his h-Pawn by allowing a Rook exchange: $59 . \mathrm{Rb} 3$ Rh1 60. Kg3 Rg1 61. Kh3 Rg4 62. Rg3 Rg3 63. Kg3 Kd6.

\section{Rb6 Bd5}

White now has to play either $60 . \mathrm{Rb} 4$ or $60 . \mathrm{Rb} 3$ to try to hold his h-Pawn. Belle thinks we are now up two full (though possibly virtual) Pawns, although it is not $100 \%$ clear to me how we win the second Pawn. I can understand it feels $60 . \mathrm{Rb} 3 \mathrm{Rh} 1+61 . \mathrm{Kg} 3 \mathrm{Rg} 1+62 . \mathrm{Kh} 3 \mathrm{Rg} 463 . \mathrm{Rg} 3 \mathrm{Rg} 3+64 . \mathrm{Kg} 3 \mathrm{Kd} 5$ as being able to snag the bPawn. But 60. Rb4 is not so obvious: maybe it thinks it can put the Bishop on $\mathrm{g} 4$ somehow and play Rh1? A possible sequence: $60 . \mathrm{Rb} 4 \mathrm{Kd} 661 . \mathrm{Nd} 3 \mathrm{Re} 262 . \mathrm{Kg} 1 \mathrm{Rg} 2+63$. Ke1 Bf3. Belle mentions 60 . Rb4 Re2+ 61 . $\mathrm{Kg} 3 \mathrm{~g}$ 5! forcing White to play hg because of the mating net and the two connected passed Pawns are enormous. Regardless, White is a Pawn down, his pieces don't coordinate and to boot, they look misplaced. Even if Black can't force win a Pawn, moving the Pawn to 55 can be played soon. Things look bleak for the forces of White. 60. Rb4 g5

We decided to remain flexible with the immediate push. Other moves are Kd6 and Re2+. Both these possibilities can be played next. We expect they will trade Pawns, but if $61 . \mathrm{Kg} 3 \mathrm{Re} 2$ ! forces the exchange due to the 
mating net.

\section{Rd4 Bc6}

The computer sees Black up 2.5 Pawns. That made me nervous because if that means Black wins the Knight for two Pawns we can't win! However, sample variations indicate that is not the case. Belle thinks $62 . \mathrm{Nd} 3$ is forced. If, instead, $62 . \mathrm{b} 4$ ?! $\mathrm{Rh} 1+63 . \mathrm{Kg} 3 \mathrm{~g} 4$ and $\mathrm{Rh} 3+$ followed by $\mathrm{Rh} 4$ nets the h-Pawn. White can always trade Pawns, but that only eases Black's task. Belle sees the main line as $62 . \mathrm{Nd} 3 \mathrm{Re} 2+63 . \mathrm{Kg} 1$ ! $(63 . \mathrm{Kg} 3$ ?? $\mathrm{Rg} 2+$ is mate in a couple) $\mathrm{g} 4$ followed by $\mathrm{Bf} 3$ if the Bishop is attacked. A subsequent $\mathrm{g} 3$ would complete a mating net.

\section{2. hxg5 fxg5}

The computer now sees us up a full piece. It thinks the main line is $63 . \mathrm{Kg} 3 \mathrm{Rg} 1+64 . \mathrm{Kf} 2 \mathrm{Rg} 2+$ winning the bPawn. We expect resignation within the next ten moves or sooner.

\section{Nd3 Re2+}

It should be noted that $63 . \mathrm{b} 4 ?$ ? $\mathrm{h} 4$ ! $64 . \mathrm{Kh} 3 \mathrm{Bf} 3$ ! is soon mate by Rh1. We sent the if move $64 . \mathrm{Kg} 1 \mathrm{~h} 465 . \mathrm{Kf} 1$ Rd2. White would be advised to accept this as we sent an error of $65 . . . \mathrm{Rd} 2 ?$ ? when 65 . ... Rc2! was our intention. White now may be able to arrange a sacrifice of his Knight for the two Pawns.

\section{Kg1 h4 65. Kf1 Rd2 66. Ke1 Rg2}

Our $65^{\text {th }}$ was a notational error $(65 . . . \mathrm{Rc} 2 !)$ that hurt our chances to win as White now has more chances to give up his Knight for the Pawns. Still, 67. Nf2 Bd7 68. Kf1 h3 69. b4 g4 looks pretty grim for the forces of White.

\section{Nf2 Rg3}

The computer preferred this to $67 . .$. Bd7 and I have no objection. Belle thinks White must play $68 . \mathrm{Rg} 4$ now or $\mathrm{Bf} 3$ and h3 follow. After 68. Rg4 Rg4 69. Ng4 Ke6 looks pretty convincing.

\section{8. b4 Bf3}

The computer thought it had analyzed $68 . . . \mathrm{h} 3$ to a win, but I didn't see what it had after $68 . . . \mathrm{h} 369$. Rg4! Re3+70. Kf1. The text prevents any such tricks and threatens to ram the h-Pawn in. White can try 68 ... Bf3 69 . $\mathrm{Rd} 3$, but $69 . . . \mathrm{g} 4$ keeps things going.

\section{Rc4 Bg2 70. b5 h3}

White cannot now play 71. Rg4 Rg4 72. Ng4 Bf3! 73. Nh2 g4! 74. Kf2 Bd1 winning. Murky is 71. Rc7+ Kf6 72. Rh7 Re3+ 73. Kd2 (73. Kf1 is not now possible!) Rb3 74. b6! Kf5 75. b7 Kf4 seemingly winning. Black threatens $\mathrm{Kg} 3$. The computer wouldn't look at such positions because it valued $\mathrm{R}+\mathrm{B}$ vs. $\mathrm{R}$ higher. The key to Black's edge is the distance of White's King from the Pawn: 76. b8 (Q) Rb8 77. Nh3+ Bh3 78. Rh3 g3 (or 78. ... Re8). Note that White can improve slightly with 76. Nh3+! Bh3 77. Rh3 Rb7 78. Ke2, but 78. ... Kg3! threatens 79 .... Rf8 sealing out the white King and 80 . Rf3 loses instantly to $80 . . . \mathrm{Rb} 2+$.

\section{1. b6 Kd8}

Belle now 'sees' the win, but unfortunately it's a draw. She felt their only chance was to try Rc7+ at their previous turn, but she didn't see the R+P vs. R endgame was [not] a win. The point of Kd8 is to stop the b-Pawn in certain lines. Rc7 now is too late (because it is not check): 71 . Rc7 h2! 72. Rh7 Re3+ 73. Kd2 Rf3 74. Ke1 Rf2 winning. If instead 71. Rb4 Kc8 72. b7+ Kb8 wins smoothly. The draw is 72. Nh3 Bh3 73. b7 Rb3 74. Rc5!! and Black cannot allow 74....g4 75. Rc8+ and must agree to halving the point. Black could have continued to play for a win by trying 70 .... Kf6.

\section{Nxh3 Bxh3}

They played the drawing line (73. Rc5! Rb3 [73.... g4? 74. b7 Rb3 75. Rc8+] 76. Rg5 Rb6 with a theoretical draw). We offered a draw with our last move.

Draw 